\title{
Prognostic value of COL6A3 in pancreatic adenocarcinoma
}

\author{
Christos Svoronos ${ }^{1}$, Georgios Tsoulfas ${ }^{2}$, Maria Souvatzi ${ }^{3}$, and Efthimios Chatzitheoklitos ${ }^{4}$ \\ ${ }^{1}$ Department of Surgery, AMEOS Klinikum, Halberstadt, Germany, ${ }^{2}$ First Department of Surgery, Aristotle University \\ of Thessaloniki, Thessaloniki, Greece, ${ }^{3}$ St Mary's Hospital Imperial College Healthcare NSH Trust, London, UK, \\ ${ }^{4}$ Unit of Pancreatic Surgery, Interbalkan Center, Thessaloniki, Greece
}

\begin{abstract}
Backgrounds/Aims: Pancreatic cancer is one of the most fatal human malignancies with poor prognosis, despite advances in therapy. Here, we evaluated the potential role of collagen type $\mathrm{VI} \alpha 3$ chain (COL6A3) as a non-invasive biomarker for pancreatic adenocarcinoma. Methods: In this study, we investigated immunohistochemically the expression of COL6A3 in 30 patients with resectable pancreatic adenocarcinoma by immunohistochemistry in a tissue sample of the cancer and a tissue sample of normal pancreas for each patient. Also, we looked for associations between COL6A3 and other prognostic factors of pancreatic cancer. Results: All of the pancreatic cancer tissue samples revealed in different ranges of intensity from weak $(+1)$ in $16.67 \%$, moderate $(+2)$ in $50 \%$, to strongly positive $(+3)$ in $33.33 \%$ staining for COL6A3. We found no moderate or strongly positive staining in normal pancreatic tissue. There was only weak positive staining in 23 samples $(76.67 \%)$ and $7(23.30 \%)$ were negative. Also, there was significant correlation between COL6A3 moderate and strongly expression and negative prognostic factors for pancreatic cancer. Conclusions: The greatest density of COL6A3 was observed in pancreatic cancer tissues and was correlated with negative prognostic factors for pancreatic cancer. Therefore, we suggest that COL6A3 could be used as prognostic factor in pancreatic cancer, but more studies need to prove its value. (Ann Hepatobiliary Pancreat Surg 2020;24:52-56)
\end{abstract}

Key Words: Pancreatic adenocarcinoma; COL6A3; Prognosis

\section{INTRODUCTION}

Pancreatic cancer is the third leading cause of cancer-related death and by 2030 , it will be the second. The five-year survival rate for pancreatic cancer is reported at $8 \%$, which is the lowest among many other common types of cancer. ${ }^{1}$ However, pancreatic cancer is difficult to diagnose early and more than $80 \%$ of pancreatic cancers are locally advanced or metastatic at the time of diagnosis. ${ }^{2}$ Identification of prognostic factors may improve the prediction of survival and selection of therapy.

The tumor microenvironment influences tumor genesis and metastasis of cancer cells. The dense stroma surrounding malignant epithelial cells is the histological hallmark of pancreatic adenocarcinoma. ${ }^{3}$ The stroma consists of numerous cellular, as well as acellular elements. The cellular components include fibroblasts, stellate cells, immune cells, endothelial cells, and nerve cells. The acel- lular compartment is comprised of extracellular matrix, which consists of collagen, fibrinogen, hyaluronan, and fibrin. ${ }^{4}$ The interactions between cancer cells and the microenvironment are complicated, as the stroma elements can provide either a support or a barrier for tumor growth or metastasis. Collagen is by far the most abundant and well-characterized component of the extracellular matrix in pancreatic cancer. ${ }^{5}$ Currently, 28 different types of collagen have been described in the extracellular matrix in the pancreas. Collagen type VI is abundantly present in pancreas and it is the predominant constituent subtype, immediately surrounding islets in the pancreas. ${ }^{6}$ Type VI collagen is structured as a trimer composed of three different alpha chains: alpha-1(VI), alpha- 2(VI), and alpha-3(VI). It is now clear that type VI collagen has a vital role and can suppress apoptosis and oxidative damage, and enhance cell growth. ${ }^{7,8}$ Many recent studies have shown the overexpression of COL6A3 in many types of

Received: October 2, 2019; Revised: November 29, 2019; Accepted: December 2, 2019

Corresponding author: Christos Svoronos

Department of Surgery, AMEOS Klinikum, Richard Wagner Strasse 65, Halbertstadt 38820, Germany

Tel: +49-15203877197, Fax: +49-3941645370, E-mail: xristos_svor@yahoo.gr

Copyright (C) 2020 by The Korean Association of Hepato-Biliary-Pancreatic Surgery

This is an Open Access article distributed under the terms of the Creative Commons Attribution Non-Commercial License (http://creativecommons.org/ licenses/by-nc/4.0) which permits unrestricted non-commercial use, distribution, and reproduction in any medium, provided the original work is properly cited. Annals of Hepato-Biliary-Pancreatic Surgery - pISSN: 2508-5778 - elSSN: 2508-5859 
cancer, which is also associated with poor prognosis in many of them, such as colon, prostate and lung cancer. ${ }^{9-11}$ However, there are few studies in the literature that have investigated the association of COL6A3 with pancreatic cancer.

The goal of this study was to investigate the expression of COL6A3 in pancreatic cancer adenocarcinoma and to compare it with the expression in the adjacent sections of pancreas where there were no cancer lesions. Furthermore, the prognostic value of COL6A3 was evaluated by comparing the expression of COL6A3 with clinicopathological prognostic factors for pancreatic cancer in these patients.

\section{MATERIALS AND METHODS}

\section{Sample collections and patients' characteristics}

Human pancreatic adenocarcinoma samples were obtained from 30 patients, who underwent a pancreaticoduodenectomy for pancreatic cancer in the Agios Dimitrios General Hospital surgical department. Also, from each patient one sample of normal pancreatic tissue was obtained without any cancer lesions. For each patient, the following information was collected: demographic data including age and sex, tumor staging, tumor characteristics, including histological type, differentiation, and infiltration of the lymph nodes, perineural, and vascular structures. Tumor staging was determined based on the AJCC Cancer Staging Manual, 7th Edition (2010). ${ }^{12}$ We also evaluated the lymph node ratio (LNR) by dividing the number of the positive lymph nodes with the number of all lymph nodes that were examined, the modified Glasgow prognostic score (mGPS) which incorporates the C-reactive protein and albumin values, the neutrophil/lymphocyte ratio (NLR), the platelet/lymphocyte ratio (PLR), and the lymphocyte/ monocyte ratio (LMR) for each patient.

\section{Immunochemistry}

Immunostaining for COL6A3 was performed on individual formalin-fixed, paraffin-embedded pancreatic adenocarcinoma and healthy pancreatic tissue sections using mouse polyclonal antibody raised against the COL6A3 (11a), (H3-2 ń sc-81766, Santa Cruz Biotechnology, Santa Cruz, CA, USA). All specimens were embedded in paraffin and cut onto glass slides as $5 \mu \mathrm{m}$ sections for immunochemistry. Slides were twice de-paraffinized using xylene solution, for 5 minutes each time. Then tissues were dehydrated by passing through descending concentrations $(100 \%, 96 \%, 80 \%, 75 \%, 70 \%)$ of alcohol and washed off with distilled water. To remove the endogenous peroxidase activity, sections were incubated with $3 \%$ hydrogen peroxide at room temperature for $30 \mathrm{~min}$. Antigen unmasking was performed in $0.01 \mathrm{M}$ sodium citrate buffer $\mathrm{pH}$ 6.1, for $15 \mathrm{~min}$ ( $\mathrm{min}$ ) at high power, according to the manufacturer's instructions. Non-specific antibody binding was blocked using Sniper, a specific blocking reagent for mouse and rabbit primary antibodies (Sniper, Biocare Medical, Concord, California, USA) for $5 \mathrm{~min}$. The sections were incubated for $1 \mathrm{~h}(\mathrm{~h})$, at room temperature, with the primary antibodies against COL6A3 diluted 1:200 in phosphate buffered saline (PBS) according to the manufacturer's instructions. The resultant immune peroxidase activity was developed using a DAB substrate kit (Vector Laboratories, California, USA) for $10 \mathrm{~min}$. Sections were counterstained with Harris' hematoxylin and mounted in Entellan (Merck, Darmstadt, Germany). Negative control sections, where the primary or secondary antibodies were omitted were also prepared.

\section{Immunohistochemistry analysis}

Immunohistochemical analyses were scored by two independent pathologists. The observers were blinded to the clinicopathological data for each specimen. Protein bands were visualized and the intensity of COL6A3 staining was scored as follows: - (negative staining), + (weak positive staining), ++ (moderate positive staining), and +++ (strong positive staining).

\section{Statistical analysis}

Statistical analysis was performed using SPSS software (SPSS standard version 16.0). Clinical characteristics were analyzed using Pearson's chi-square or Fisher's exact test for categorical and dichotomous variables, and Student's t-test for continuous variables. Statistical significance was assumed for a two-tailed $p$-value of $<0.05$.

\section{RESULTS}

\section{Study patients' characteristics}

Samples were collected from 30 patients with pancreatic adenocarcinoma. The median age was 68.2 (44-86) 
years. All the patients had resectable pancreatic tumors and underwent a pancreaticoduodenectomy. The pathologic evaluation of the surgical specimens revealed an R0 resection in all the patients of the study. Most of the patients had AJCC stage IIb (70\%) and IIa (20\%). The pancreatic adenocarcinoma was moderately- and poorly-differentiated in $11(36.67 \%)$ and $19(63.33 \%)$ patients, respectively. Perineural invasion was identified in 19 (63.33\%) patients, whereas vascular invasion was found in $21(70 \%)$ patients. The clinicopathologic characteristics of the patients in this study are summarized in Table 1.

Table 1. Clinicopatholigical characteristics of the cohort patients $(\mathrm{n}=30)$

\begin{tabular}{lc}
\hline Age $(\mathrm{y})$ & $68.2(44-86)$ \\
Sex (male/female) & $18(60 \%) / 12(40 \%)$ \\
AJCC stage & $1(3.33 \%)$ \\
Ia & $2(6.67 \%)$ \\
Ib & $6(20 \%)$ \\
IIa & $21(70 \%)$ \\
IIb & \\
Tumor differentiation & 0 \\
G1 & $11(36.67 \%)$ \\
G2 & $19(63.33 \%)$ \\
G3 & $9(30 \%)$ \\
N Status & $21(70 \%)$ \\
NO & $19(63.33 \%)$ \\
N1 & $11(36.67 \%)$ \\
Perineural invasion & \\
Positive & $21(70 \%)$ \\
Negative & $9(30 \%)$ \\
Microvascular invasion & \\
Positive &
\end{tabular}

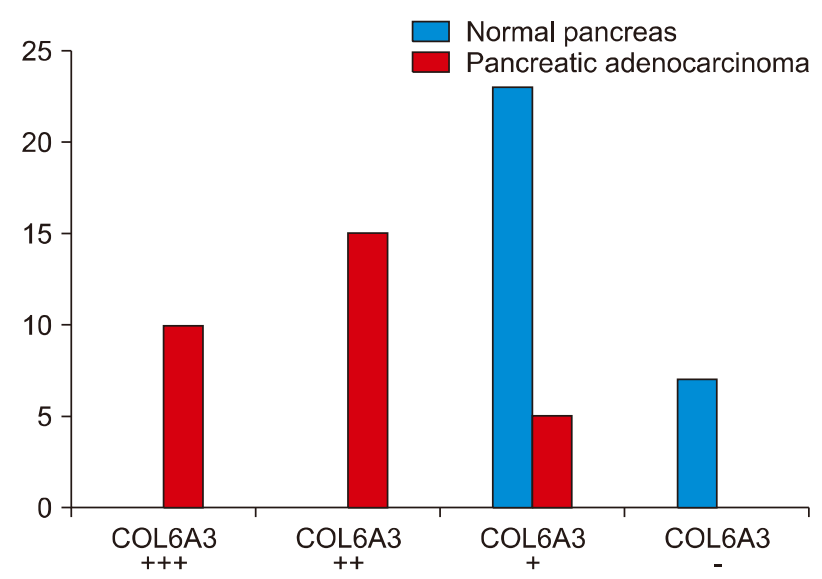

Fig. 1. Immunochistochemical expression of COL6A3 in pancreatic adenocarcinoma and pancreatic healthy tissue.

\section{Expression of COL6A3 protein}

According to the protein band intensity in the stroma regions, the expression of COL6A3 in the stroma of pancreatic adenocarcinoma was strong positive, moderate positive and weak positive in $10(33.33 \%), 15(50 \%)$, and $5(16.67 \%)$ samples, respectively. Among the normal pancreatic tissue samples the expression of COL6A3 was negative and weak positive in 23 (76.67\%) and 7 (23.33\%) samples, respectively. There was no strong or moderate positive expression of COL6A3 observed in normal pancreatic tissue (Fig. 1).

\section{COL6A3 expression is associated with prognostic factors of pancreatic cancer}

In order to evaluate the impact of COL6A3 expression on prognosis of pancreatic cancer, we evaluated the association between the positive expressions of COL6A3 and other prognostic factors of pancreatic cancer. Among the prognostic factors, which were evaluated in this study,

Table 2. Strong or moderate positive expression of COL6A3 and prognostic factor of pancreatic cancer

\begin{tabular}{|c|c|c|}
\hline & $\begin{array}{l}\text { COL6A3+++/ } \\
\text { COLA6A3++ }\end{array}$ & $p$-value \\
\hline \multicolumn{3}{|l|}{ Tumor differentiation } \\
\hline G2 & $8(32 \%)$ & 0.012 \\
\hline G3 & $17(68 \%)$ & \\
\hline \multicolumn{3}{|l|}{ Lymph node metastasis } \\
\hline Yes & $19(76 \%)$ & 0.01 \\
\hline No & $6(24 \%)$ & \\
\hline Lymph node ratio $>0.3$ & $14(56 \%)$ & 0.56 \\
\hline Lymph node ratio $<0.3$ & $11(44 \%)$ & \\
\hline CA $19-9>100 \mathrm{u} / \mathrm{ml}$ & $12(48 \%)$ & 0.61 \\
\hline CA $19-9<00 \mathrm{u} / \mathrm{ml}$ & $13(52 \%)$ & \\
\hline $\mathrm{CEA}>10 \mathrm{mg} / \mathrm{dl}$ & $5(20 \%)$ & $<0.001$ \\
\hline $\mathrm{CEA}<10 \mathrm{mg} / \mathrm{dl}$ & $20(80 \%)$ & \\
\hline mGPS 0 & $15(60 \%)$ & 0.52 \\
\hline mGPS 1,2 & $10(40 \%)$ & \\
\hline Neutrophil/lymphocyte ratio $>5$ & $19(76 \%)$ & 0.01 \\
\hline Neutrophil/lymphocyte ratio $<5$ & $6(24 \%)$ & \\
\hline Platelet/lymphocyte ratio $>160$ & $10(60 \%)$ & 0.52 \\
\hline Platelet/lymphocyte ratio $<160$ & $15(40 \%)$ & \\
\hline Lymphocyte/monocyte ratio $<3$ & $8(32 \%)$ & 0.012 \\
\hline Lymphocyte/monocyte ratio $<3$ & $17(68 \%)$ & \\
\hline \multicolumn{3}{|l|}{ Perineural invasion } \\
\hline Yes & $17(68 \%)$ & 0.012 \\
\hline No & $8(32 \%)$ & \\
\hline \multicolumn{3}{|l|}{ Microvascular invasion } \\
\hline Yes & $20(80 \%)$ & $<0.001$ \\
\hline No & $5(20 \%)$ & \\
\hline
\end{tabular}


lymph node ratio $>0.3(p=0.56)$, CA 19-9 $>100 \mathrm{u} / \mathrm{ml}(p=$ $0.61), \mathrm{CEA}>10 \mathrm{mg} / \mathrm{dl}, \mathrm{mGPS}$, and platelet/lymphocyte ratio $(p=0.52)$ had no statistically significant association with the strong positive or moderate positive expression of COL6A3. In contrast, tumor differentiation $(p=0.012)$, lymph node metastasis $(p=0.01)$, neutrophil/lymphocyte ratio $>5(p=0.01)$, lymphocyte/monocyte ratio $<3 \quad(p=0.012)$, perineural invasion $(p=0.012)$, and microvascular invasion $(p<0.001)$ were associated with strong or moderate positive expression of COL6A3 (Table 2).

\section{DISCUSSION}

Pancreatic adenocarcinoma remains a lethal disease with very poor prognosis. Although surgery is the only curative treatment option, the 5-year survival, even after curative resection, is still only $15-25 \% .^{13,14}$ Multiple previous studies have demonstrated that clinicopathologic factors such as tumor stage, histological differentiation, nodal involvement, perineural invasion and microvascular invasion are statistically significant prognostic variables. ${ }^{15,16}$ Also, numerous studies have shown the prognostic value of LNR in pancreatic cancer. ${ }^{17,18}$ Furthermore, various inflammatory markers, as mGPS, NLR, PLR, and LMR have been shown to be significant prognostic factors of the disease. ${ }^{19-22}$ Nevertheless, the number of patients alive at 5 years makes these prognostic variables only marginally influential. Therefore, it is important to understand the intrinsic properties of the development and the progression of the pancreatic cancer and to identify more accurate prognostic factors for more effective therapies.

The COL6A3 is an extracellular matrix protein, which is usually found in most connective tissues, including muscle, skin, tendon, and vessels. Recently, many studies revealed the important role of COL6A3 in the diagnosis and prognosis of colorectal, lung and prostate cancer. First, Arafat et al. ${ }^{23}$ in 2011, compared ,using Western blot analysis and immunochemistry, 8 specimens of pancreatic adenocarcinoma with premalignant lesions and normal pancreas, and showed a high expression of COL6A3 in pancreatic adenocarcinoma and that a dynamic process of alternative splicing of the COL6A3 gene is exclusively associated with malignancy. Another study, from Kang et al. ${ }^{24}$ at 2014, comparing the serum levels of COL6A3 in patients with pancreatic cancer and pa- tients with benign lesions and healthy people, revealed that COL6A3 protein levels were significantly elevated in patients with pancreatic cancer and demonstrated the potential clinical significance of circulating COL6A3 in the diagnosis of pancreatic cancer.

In the present study, we investigated the expression of COL6A3 in pancreatic adenocarcinoma and compared it with the expression of COL6A3 in normal pancreas of the same patients. Our immunohistochemical analysis revealed that COL6A3 was predominantly expressed in the pancreatic cancer stroma. This difference highlights the important role of COL6A3 in the microenvironment during the development and progression of pancreatic cancer. Also, we found that high expression of COL6A3 was associated with most of the negative prognostic factors for pancreatic cancer. Specifically, most of the patients with strong or moderate positive expression of COL6A3 had poor differentiated tumors, lymph node metastasis by the diagnosis, NLR $>5$, LMR $<3 \quad(p=0.012)$, and perineural and microvascular invasion. These results reflect that overexpression of COL6A3 is associated with poor prognosis of pancreatic cancer and suggest that COL6A3 could be used as a prognostic factor in pancreatic adenocarcinoma.

In summary, our results demonstrate that COL6A3 is overexpressed in pancreatic cancer stroma, suggesting that COL6A3 is involved in the progress of pancreatic cancer. Also, COL6A3 was associated with negative prognostic factors of the disease, indicating that it could be a useful prognostic factor for pancreatic adenocarcinoma. In order to verify these results, a larger sample size and more in-depth analyses will be needed.

\section{ORCID}

Christos Svoronos: https://orcid.org/0000-0002-6375-6134

Georgios Tsoulfas: https://orcid.org/0000-0001-5043-7962

Maria Souvatzi: https://orcid.org/0000-0002-5269-5671

Efthimios Chatzitheoklitos: https://orcid.org/0000-00029912-5190

\section{AUTHOR CONTRIBUTIONS}

Conceptualization: Christos Svoronos, Georgios Tsoulfas. Data curation: Christos Svoronos, Georgios Tsoulfas, Efthimios Chatzitheoklitos. Formal analysis: Christos Svoronos, Georgios 
Tsoulfas, Efthimios Chatzitheoklitos, Maria Souvatzi. Methodology: Christos Svoronos, Georgios Tsoulfas. Project administration: Christos Svoronos, Georgios Tsoulfas, Efthimios Chatzitheoklitos. Visualization: Christos Svoronos, Georgios Tsoulfas. Writing - original draft: Christos Svoronos. Writing - review \& editing: Christos Svoronos, Georgios Tsoulfas, Efthimios Chatzitheoklitos, Maria Souvatzi.

\section{REFERENCES}

1. Siegel RL, Miller KD, Jemal A. Cancer Statistics, 2017. CA Cancer J Clin 2017;67:7-30.

2. Jemal A, Bray F, Center MM, Ferlay J, Ward E, Forman D. Global cancer statistics. CA Cancer J Clin 2011;61:69-90.

3. Rucki AA, Zheng L. Pancreatic cancer stroma: understanding biology leads to new therapeutic strategies. World J Gastroenterol 2014;20:2237-2246.

4. Neesse A, Bauer CA, Öhlund D, Lauth M, Buchholz M, Michl $\mathrm{P}$, et al. Stromal biology and therapy in pancreatic cancer: ready for clinical translation? Gut 2019;68:159-171.

5. Weniger M, Honselmann KC, Liss AS. The extracellular matrix and pancreatic cancer: a complex relationship. Cancers (Basel) 2018;10:E316.

6. Llacua LA, Hoek A, de Haan BJ, de Vos P. Collagen type VI interaction improves human islet survival in immunoisolating microcapsules for treatment of diabetes. Islets 2018;10:60-68.

7. Zazuli Z, Barliana MI, Mulyani UA, Perwitasari DA, Ng H, Abdulah R. Polymorphism of PXR gene associated with the increased risk of drug-induced liver injury in Indonesian pulmonary tuberculosis patients. J Clin Pharm Ther 2015;40:680-684.

8. Moon JY, Chang BC, Lee KE, Bang JS, Gwak HS. Effects of pregnane $\mathrm{X}$ receptor genetic polymorphisms on stable warfarin doses. J Cardiovasc Pharmacol Ther 2015;20:532-538.

9. Liu W, Li L, Ye H, Tao H, He H. Role of COL6A3 in colorectal cancer. Oncol Rep 2018;39:2527-2536.

10. Duan Y, Liu G, Sun Y, Wu J, Xiong Z, Jin T, et al. COL6A3 polymorphisms were associated with lung cancer risk in a Chinese population. Respir Res 2019;20:143.

11. Xie X, Liu X, Zhang Q, Yu J. Overexpression of collagen VI $\alpha 3$ in gastric cancer. Oncol Lett 2014;7:1537-1543.

12. American Cancer Society. American Joint Committee on Cancer (AJCC) TNM staging system. New York: American Cancer Society; 2016 [cited 2017 Jan 4]. Available from: http://www.cancer.org/ cancer/pancreaticcancer/detailedguide/pancreatic-cancer-staging.

13. Lin R, Han CQ, Wang WJ, Liu J, Qian W, Ding Z, et al. Analysis on survival and prognostic factors in patients with resectable pancreatic adenocarcinoma. J Huazhong Univ Sci Technolog Med Sci 2017;37:612-620.

14. Yamamoto T, Yagi S, Kinoshita H, Sakamoto Y, Okada K, Uryuhara $\mathrm{K}$, et al. Long-term survival after resection of pancreatic cancer: a single-center retrospective analysis. World $\mathrm{J}$ Gastroenterol 2015;21:262-268.

15. Vernerey D, Huguet F, Vienot A, Goldstein D, Paget-Bailly S, Van Laethem JL, et al. Prognostic nomogram and score to predict overall survival in locally advanced untreated pancreatic cancer (PROLAP). Br J Cancer 2016;115:281-289.

16. Wang XD, Qian JJ, Bai DS, Li ZN, Jiang GQ, Yao J. Marital status independently predicts pancreatic cancer survival in patients treated with surgical resection: an analysis of the SEER database. Oncotarget 2016;7:24880-24887.

17. Butturini G, Stocken DD, Wente MN, Jeekel H, Klinkenbijl JH, Bakkevold KE, et al.; Pancreatic Cancer Meta-Analysis Group. Influence of resection margins and treatment on survival in patients with pancreatic cancer: meta-analysis of randomized controlled trials. Arch Surg 2008;143:75-83; discussion 83.

18. Pawlik TM, Gleisner AL, Cameron JL, Winter JM, Assumpcao $\mathrm{L}$, Lillemoe KD, et al. Prognostic relevance of lymph node ratio following pancreaticoduodenectomy for pancreatic cancer. Surgery 2007; 141:610-618.

19. Asari S, Matsumoto I, Toyama H, Shinzeki M, Goto T, Ishida $\mathrm{J}$, et al. Preoperative independent prognostic factors in patients with borderline resectable pancreatic ductal adenocarcinoma following curative resection: the neutrophil-lymphocyte and platelet-lymphocyte ratios. Surg Today 2016;46:583-592.

20. Yu J, Ding Z, Yang Y, Liu S. Increased platelet-to-lymphocytes ratio is associated with poor long-term prognosis in patients with pancreatic cancer after surgery. Medicine (Baltimore) 2018;97: e11002.

21. Li W, Tao L, Zhang L, Xiu D. Prognostic role of lymphocyte to monocyte ratio for patients with pancreatic cancer: a systematic review and meta-analysis. Onco Targets Ther 2017;10:33913397.

22. La Torre M, Nigri G, Cavallini M, Mercantini P, Ziparo V, Ramacciato G. The glasgow prognostic score as a predictor of survival in patients with potentially resectable pancreatic adenocarcinoma. Ann Surg Oncol 2012;19:2917-2923.

23. Arafat H, Lazar M, Salem K, Chipitsyna G, Gong Q, Pan TC, et al. Tumor-specific expression and alternative splicing of the COL6A3 gene in pancreatic cancer. Surgery 2011;150:306-315.

24. Kang CY, Wang J, Axell-House D, Soni P, Chu ML, Chipitsyna $\mathrm{G}$, et al. Clinical significance of serum COL6A3 in pancreatic ductal adenocarcinoma. J Gastrointest Surg 2014;18:7-15. 